\title{
Theoretical Analysis of Possible Martensitic Phases in Titanium Nickelide
}

\author{
V.E. Naish, T.V. Novoselova and I.V. Sagaradze \\ Institute of Metal Physics, Kovalevskaya Str. 18, 620219 Ekaterinburg, Russia
}

\begin{abstract}
The concept of co-operetive thermal vibrations in crystals is applied here for an independent analysis of a system of martensitic phase transitions in titanium nickelide (NiTi). In addition to the orthorhombic B19 phase, which is well known for NiTi, a trigonal and a monoclinic phase - have been produced and are analyzed in detail. The calculated and experimental neutron diffraction patterns obtained for monoclinic and trigonal phases show a good agreement. The obtained structure of the monoclinic phase is absolutely different from the B19' structure described in the literature. Finally. more emphasis is placed on some complicated problems associated with a detailed identification of the trigonal phase structure.
\end{abstract}

\section{INTRODUCTION}

The reconstruction of the various crystal structures under the structural phase transitions of the cooperative (martensitic) type is described in many our articles on the basis of concept of co-operative thermal vibrations with their transformation into static displacements. The generalization of these works is given in our review [1].

According to this concept there are the extended one- and two-dimensional coherent objects in crystals, i.e. atomic chains or planes in crystals inside which the atoms vibrate with considerable correlation so as a coherency in the object is conserved. At elevated temperatures (above the transition point) coopereative vibrations determine complex figures of thermal diffuse scattering, which are observed in the $\mathrm{X}$ ray mono-Laue experiment (and also on electron diffraction patterns). As the temperature is lowered, cooperative vibrations of the objects from one or several crystallographically equivalent families freeze. This underlies the mechanism of a spontaneous structural phase transition of the co-operative (martensitic) type. It is discussed here an application of this theory to NiTi crystals.

At high temperature NiTi possesses a crystal structure of the $\mathrm{B} 2$ type (or $\mathrm{CsCl}$ ). Under different conditions three types of martensitic phases - orthorhombic B19, monoclinic B19', and rhombohedral $\mathrm{R}$ phase - can be formed on cooling NiTi. Sometimes a consecutive alternation of these phases is observed. As far as we know, up to now there has been no theory to account for these transitions based on a unified concept. The goal of the present work is to construct such theory.

Freezing of the vibrations means that the coherent extended object remains deflected with a shift $\Delta$. Naturally, gaps appear between the atoms-spheres that is energetically unfavorable. Therefore, the crystal structure must transform to more close-packed one. This process is conceptually an uniform deformation and is called a contraction. So, to describe the entire system of the atom movements during a phase transition, we have to introduce three more quantities - three components of the contraction (intra-object $k_{1}$ and inter-object $k_{\|}$and $k_{z}$ ) - in addition to the object shift $\Delta$

Independent crystallochemical analyses showed that in the B2 structure the only possible extended coherent objects are atomic planes of the (110) type. These objects-planes are capable of moving only along themselves in the $[1 T 0]$ direction. The cubic B2 structure comprises six families of such planes. As the temperature is lowered, in the general case vibrations of several families at the same time or of one of the families can freeze at the first transition point. Several a priori possible alternatives correspond to several different structures of the martensitic phases produced. It is convenient to introduce the multicomponent order parameter $\eta$ with the components $\eta_{\alpha}$ corresponding to each $\alpha$-th family of objects: 


$$
\eta=C_{1} \eta_{x y}+C_{2} \eta_{\bar{x} y}+C_{3} \eta_{y z}+C_{4} \eta_{\bar{y} z}+C_{5} \eta_{z x}+C_{6} \eta_{\bar{z} x}
$$

As it is well known from the general theory of phase transitions, the most probable new phases are those that correspond to the selected combinations of the mixing coefficients $C_{\alpha}$. The first one is the version (0C0000), where vibrations of only one $\bar{x} y$-family freeze. Secondly, let us consider the (0C000C) version with simultaneous participation of two families, $\bar{x} y$ and $\bar{z} x$. This pair intersect one another in the lines of the [111] type. Finally, the three planes $\bar{x} y, \bar{y} z$ and $\bar{z} x$ intersecting in the same line [111] obviously deserve attention. This is the version $(0 \mathrm{COC} 0 \mathrm{C})$. We shall not consider more complicated combinations since the probability of realizing the corresponding phases is much lower.

Freezing of the vibrations of the objects-planes means transformation of their dynamic displacements into static ones. This process can proceed by two different mechanisms. First, the mutual displacement of successive pairs of the planes takes the same direction and the shear accumulates. This is the shear mechanism. Second, the shear is compensated at every consecutive interplanar spacing. This is the compensation mechanism.

It is convenient to describe displacements of the planes using the simplest version of the theory, namely in the terms of the Ising's model, where vibrations of an object about the equilibrium position are treated as jumps from the "left-hand" position to the "right-hand" one. Then, two possible mechanisms for freezing of the planes correspond to the two different signs of the Ising parameter $V$ of interaction between the displacements of neighboring planes.

Naturally, only one freezing mechanism holds for a given material. Namely, the compensation mechanism is appropriate in NiTi only. This situation is illustrated schematically on Fig. 1.

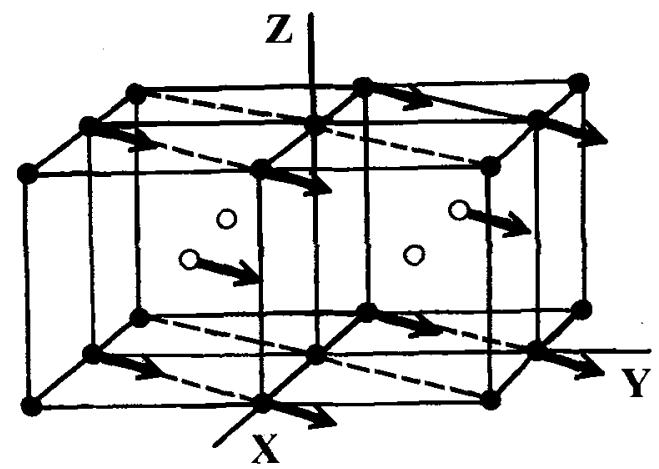

Figure 1: Relative displacements of atoms forming the objects-planes with the compensation mechanism for B2 structure. $\Leftrightarrow)$. All atoms in middle plane account as immovable than all arrows $(\Rightarrow)$ shown the relative displacements of all other planes.

\section{A SCHEME FOR CALCULATION OF THE MARTENSITIC PHASE STRUCTURE}

For this case detailed analysis of the structures of new phases was fulfilled in our work [2]. This analysis includes a few stages.

At first, one must write the shifted coordinates of atoms accounting for the plane shears $\Delta$ and the contraction displacements $k_{\|}, k_{\perp}$ and $k_{z}$. All those values are convenient to describe by the notion of the relative radius $\mathrm{r}=\mathbf{R}_{\mathrm{N} /} / \mathrm{R}_{\mathrm{Ti}}$.

Further one must write the exact expressions for the differences of atomic coordinates of many atomic pairs in order to examine which translations are preserved in the new phase. Then we can find three shortest preserved translations and construct the cell of new phase. This means that a set of exact atomic coordinates for all atoms inside the new cell is a solution of the problem. Further it is possible to determine a space group of the structure of new phase. 
The numerical values of parameters $\Delta, \mathrm{k}_{\|,}, \mathrm{k}_{\perp}, \mathrm{k}_{\mathrm{z}}$ (which define the exact atomic coordinates) must be determined with the help of the usual full-profile analysis of the calculated and experimental intensities of the reflections on the $\mathrm{X}$-ray or neutron diffraction patterns.

\section{RESULTS}

\subsection{The orthorhombic phase structure}

The use of the first mixing variant $(0 \mathrm{C} 0000)$ gives the orthorhombic structure which precisely coincides with wellknown structure B 19 for $\mathrm{NiTi}$. So, we state an agreement between all the calculated data and the experimental ones. All details of our calculations are described in reference [2].

\subsection{The monoclinic phase structure}

An analysis of the second mixing variant $(0 \mathrm{C} 000 \mathrm{C})$ leads to a monoclinic structure with a space group $\mathrm{Bm}=\mathrm{C}_{\mathrm{s}}{ }^{3}$. As known from the literature, a monoclinic martensitic phase B 19' does exist for NiTi. But its monoclinic cell is absolutely different from proposed one. It has quite different dimensions, is oriented differently relative to the initial cubic B2 lattice, and comprises much less atoms. Detailed description of this monoclinic martensitic structure is published in our paper [2] (see its primitive cell on Fig.2). But we say some words about it here.

The orientation relations between initial and new lattice are shown on Fig. 2 too

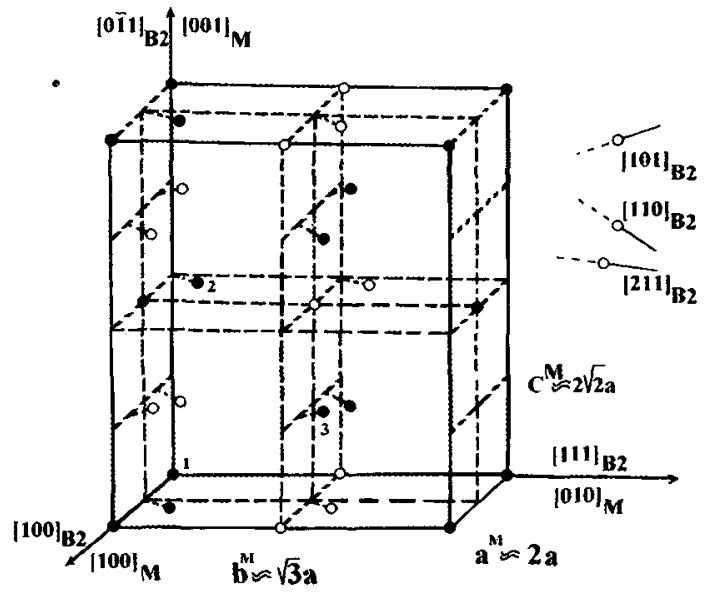

Figure 2: A base-centered unit cell of the monoclinic phasc with its oricntation relativcly to the initial B2 sinuclure. Diffcrent atoms $\mathrm{Ni}(\bullet)$ and $\mathrm{Ti}(\mathrm{o})$ are shown in accordance with the set of their coordinates.

The monoclinic coordinates of $4 \mathrm{Ni}$ atoms are:

1. $0,0,0$

2. $2 \mathrm{x}, 2 \mathrm{y}, 1 / 2$

3. $3 / 4+x, 1 / 2+y, 1 / 4+z$

4. $1 / 4+x, 1 / 2+y, 1 / 4-z$

The coordinates of 4 different $\mathrm{Ni}$ atoms can be obtained from these by adding the vector $(1 / 2,0,1 / 2)$. In its turn the coordinates of all $8 \mathrm{Ti}$ atoms can be obtained from the coordinates of $8 \mathrm{Ni}$ atoms by adding the vector $(0,1 / 2,0)$.

The exact values, obtained by fitting our model to experimental neutron diffraction pattern [3] are: 


$$
\begin{aligned}
& \mathrm{a}=5.870 \stackrel{\circ}{A}, \quad \mathrm{~b}=5.957 \stackrel{\circ}{A}, \quad \mathrm{c}=8.743 \stackrel{\circ}{A}, \quad \gamma=45.9^{\circ} \\
& \mathrm{x}=0.027, \quad \mathrm{y}=0.015, \quad \mathrm{z}=0.03 \text {. }
\end{aligned}
$$

So, we have to admit a sharp disagreement between the obtained results and the literature data. However, the proposed a new model for the structure of the monoclinic phase in NiTi which agrees with experimental findings [3] with an agreement not worse than in the case of the stereotype model B19'. Imperfect agreement between our theory and that experiment due to the different relative intensities of the reflections is explained by existence of the texture in that specimen of NiTi.

\subsection{The rhombohedral phase structure 3.3. I In compensation mechanism}

At last, consideration of a third mixing type $(0 \mathrm{COCOC})$ in the same compensation mechanism gives any rhombohedral structure with the space group $\mathrm{C}_{3 v}{ }^{5}=\mathrm{R} 3 \mathrm{~m}$. The least cell is rhombohedr here. The total description of this structure is also printed in the paper [2]. It is possible to describe this structure in the hexagonal axes; then the obtained edges of hexagonal cell are $a^{\mathrm{H}}=a \sqrt{8}$ and $c^{\mathrm{H}}=a \sqrt{3}$ (where $a$ is the lattice parameter of initial cubic phase), just as the edge $\mathrm{a}^{\mathrm{H}}$ in NiTi must be equal $\sim \mathrm{a} \sqrt{6}$. Besides we have in such way the superstructure reflections of type $1 / 2 \quad 1 / 20$ just as they must be of type $1 / 31 / 30$. Therefore our compensation mechanism is not suitable to explain the right structure of R-phase in NiTi. The obtained rhombohedral structure exists in some other crystals, probably for $\mathrm{NaHg}$ [4]

\subsubsection{In alternative mechanism}

It is necessary to use another mechanism instead of compensation for description the R-phase of NiTi. To do this we must use the Ising model with the interaction of the nearest $V$ and second $V$ ' neighbors and we will assume $V^{\prime}$ as sufficiently large. This opens the possibility for the more complex configurations of the plane shifts under freezing. The period of the configurations can be more than 1 as in shear mechanism or 2 as in compensation mechanism. Let us assume it to equal 3: two planes are shifted in the same side by $\Delta$, third plane is immovable etc. It is necessary to carry out all computations once again: the edges of new cell, coordinates of all atoms, the space group of new phase etc. Here we can give only the final results of such computations, while the detailed description of all calculation procedure is in our article [5].

Three shortest non-complanar preserved translations are approximately equal

$$
\vec{a}_{1}-2 \vec{a}_{2}+\vec{a}_{3}, \quad \vec{a}_{1}+\vec{a}_{2}-2 \vec{a}_{3}, \quad \vec{a}_{1}+\vec{a}_{2}+\vec{a}_{3}
$$

We designate them $a_{1}^{H}, a_{2}^{H}, a_{3}^{H}$ and write their exact components in cubic axes which account for all atoms displacements:

$$
\begin{aligned}
& \vec{a}_{1}^{H}=\left(a+k_{\|}-3 k_{\perp}-2 k_{z} ;-2 a-2 k_{\|}+6 k_{\perp}+4 k_{z} ; a+k_{\|}-3 k_{\perp}-2 k_{z}\right) ; \\
& \vec{a}_{2}^{H}=\left(a+k_{\|}-3 k_{\perp}-2 k_{z} ; a+k_{\|}-3 k_{\perp}-2 k_{z} ;-2 a-2 k_{\|}+6 k_{\perp}+4 k_{z}\right) ; \\
& \vec{a}_{3}^{H}=\left(a+4 k_{\|}-2 k_{z} ; a+4 k_{\|}-2 k_{z} ; a+4 k_{\|}-2 k_{z}\right) .
\end{aligned}
$$

It can be shown that the angles $\left(\overrightarrow{\mathrm{a}}_{1}^{\mathrm{H}}, \overrightarrow{\mathrm{a}}_{3}^{\mathrm{H}}\right)$ and $\left(\overrightarrow{\mathrm{a}}_{2}^{\mathrm{H}}, \overrightarrow{\mathrm{a}}_{3}^{\mathrm{H}}\right)$ are equal $90^{\circ}$ and the angle $\left(\overrightarrow{\mathrm{a}}_{1} \hat{\mathrm{H}}_{\overrightarrow{\mathrm{a}}} \mathrm{H}_{2}\right)$ is equal $120^{\circ}$. Thus the primitive cell is hexagonal. The approximate values of the cell edges are $\mathrm{a}=\mathrm{H} \sqrt{6}$ and $\mathrm{c}^{\mathrm{H}} \approx \mathrm{a} \sqrt{3}$. It corresponds to experimental situation in $\mathrm{NiTi}$. The exact values of edges are equal:

$$
\mathrm{a}^{\mathrm{H}}=\left(\mathrm{a}+\mathrm{k}_{\|}-3 \mathrm{k}_{\perp}-2 \mathrm{k}_{\mathrm{z}}\right) \sqrt{6} ; \quad \mathrm{c}^{\mathrm{H}}=\left(\mathrm{a}+4 \mathrm{k}_{\|}-2 \mathrm{k}_{\mathrm{z}}\right) \sqrt{3} .
$$

The orientation relations between initial and new lattices are as following:

$$
{ }^{(001)_{H}}\left\|(111)_{B 2} ; \quad[100]_{H}\right\|[1 \overline{2} 1]_{B 2} ; \quad[010]_{H} \|[11 \overline{2}]_{B 2}
$$

A volume of the hexagonal cell approximately equals $27 \mathrm{a}^{3}$. So, the hexagonal cell contains $27 \mathrm{Ni}$ atoms and $27 \mathrm{Ti}$ atoms. It is sufficiently to consider only $9 \mathrm{Ni}$ atoms and $9 \mathrm{Ti}$ atoms belonging to the primitive cell which is $1 / 3$ of hexagonal cell. The hexagonal coordinates of these $9 \mathrm{Ni}$ atoms are: 
1. $0 ; 0 ; 0$

2. $1 / 3-\mathrm{u}-\mathrm{v} ; 0 ; 2 / 3+4 \delta$

3. $0 ; 1 / 3-\mathrm{u}-\mathrm{v} ; 2 / 3+4 \delta$

4. $2 / 3+u+v ; 2 / 3+u+v ; 2 / 3+4 \delta$

5. $2 / 3-u+v ; 0 ; 1 / 3+4 \delta$

6. $0 ; 2 / 3-u+v ; 1 / 3+4 \delta$

7. $1 / 3+u-v ; 1 / 3+u-v ; 1 / 3+4 \delta$

8. $2 / 3 ; 1 / 3 ; 6 \delta$

9. $1 / 3 ; 2 / 3 ; 6 \delta$

The coordinates of the $9 \mathrm{Ti}$ atoms can be obtained from them by adding the vector $(001 / 2)$. Here the quantities $u, v, \delta$ are expressed by the relations:

$$
\mathrm{u}=\frac{\Delta}{3\left(\mathrm{a}+\mathrm{k}_{\|}-3 \mathrm{k}_{\perp}-2 \mathrm{k}_{\mathrm{z}}\right)} ; \quad \mathrm{v}=\frac{\mathrm{p}}{\mathrm{a}+\mathrm{k}_{\|}-3 \mathrm{k}_{\perp}-2 \mathrm{k}_{\mathrm{z}}} ; \quad \delta=\frac{\Delta}{3\left(\mathrm{a}+4 \mathrm{k}_{\|}-2 \mathrm{k}_{\mathrm{z}}\right)} ;
$$

where $\mathrm{p}$ - is a magnitude denoting the space between two neighboring planes. They are small quantities of the order $\sim \Delta / \mathrm{a}$ or $\mathrm{k}_{\mathrm{i}} / \mathrm{a}$. Fig. 3 illustrates the arrangement of the hexagonal cell in view of the projection on the plane $(001)_{\mathrm{H}}$. It is evident that this structure has the symmetry axis of $3 \mathrm{~d}$ order and the family of three mirror planes $\mathrm{m}$ which pass by translations $\mathrm{a}_{1}{ }^{\mathrm{H}}$ and $\mathrm{a}_{2}{ }^{\mathrm{H}}$. The inversion is absent here. Thus a space group $\mathrm{C}_{3 \mathrm{~V}}^{2}=\mathrm{P} 31 \mathrm{~m}$.

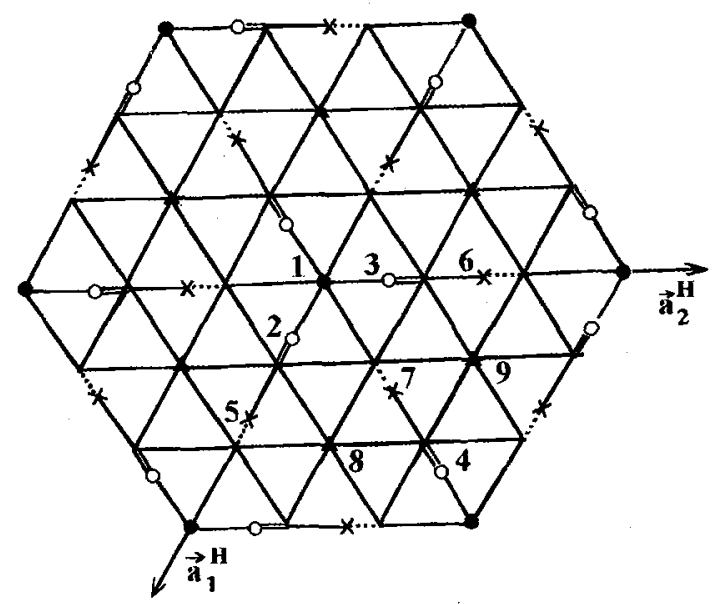

Figure 3: A projection of the hexagonal unit cell on the plane $(001)_{H}$ for trigonal martensitic phase. Only atoms $B$ are shown as atoms $A$ are situated exactly above atoms $B$ by $1 / 2 \mathrm{c}^{\mathrm{H}}$. Atoms marked by different symbols are located at different heights above the cell bottom, namely: $\mathrm{z}^{\mathrm{H}}=0$ for atoms $\ell, \quad \mathrm{z}^{\mathrm{H}}=6 \delta$ for atoms $; ; \mathrm{z}^{\mathrm{H}}=1 / 3+4 \delta$ for atoms $\square ; z^{\mathrm{H}}=2 / 3+4 \delta$ for atoms $\mp$. Horizontal displacements of atoms from the rational positions are equal to $\mathrm{u}+\mathrm{v}(\Longrightarrow)$ and $\mathrm{u}-\mathrm{v}(\cdots)$.

It is necessary to note that similar picture have appeared already in the literature, namely the same atomic arrangement of cell have been suggested in the paper [6] for AuCd. However authors of [6]on the basis of single-crystal X-Ray pattern treatment have found the positions of atoms in threes (f.e. 2, 3, and 4, or 5, 6 and 7) deviated enough from their rational ones of type 1/3. This means that despite the obvious presence of the mirror planes $m_{\|}$in the picture for AuCd their absence is ranked to be proved. So authors of [6] proposed with confidence the space group P3 and the positions of atoms like ours. 


\subsection{A comparison with the experimental data}

The chosen mechanism (more complicated than compensation one) guarantees the necessary superstructure reflections of type $1 / 31 / 30$ which observed in X-ray or neutron diffraction patterns. In our geometry it is easily shown that reflection $(100)_{\mathrm{H}}$ corresponds to $1 / 3(110)_{\mathrm{B} 2}$, reflection $(110)_{\mathrm{H}}$ corresponds to $1 / 3(211)_{\mathrm{B} 2}$ and reflection $(001)_{\mathrm{H}}$ corresponds to $1 / 3(111)_{\mathrm{B} 2}$.

Authors [7] carried out their experiment on a specimen $\mathrm{Ti}_{50} \mathrm{Ni}_{47} \mathrm{Fe}_{3}$ and they declared a space group as $\mathrm{P} \overline{3} \mathrm{~lm}$ for it. Although in [7] only three peaks were presented we have made an attempt to fit them. A good agreement over the angles and intensities have been received with following values of the structure parameters:

$$
\begin{aligned}
& \mathrm{a}^{\mathrm{H}}=7,319 \AA . \circ, \quad \mathrm{c}^{\mathrm{H}}=5,277 \AA \\
& \delta=0,013, \quad \mathrm{u}=0,013, \quad \mathrm{v}=0,008 .
\end{aligned}
$$

Unfortunately there is only the view of X-ray pattern in [8] and the detailed numerical data are absent. But a satisfactory agreement with the experimental data has been gained using just the same values as written above. We must say some words about specific reflex (202) which is mentioned in [8] as characteristic for P3 group and nonexistent for $P \overline{3} \mathrm{~lm}$. But it is well known that such symmetry elements as mirror planes (in contrast to glide planes or screw axes) can not cause the systematic absences of any reflections. In fact calculations in our model show the presence of (202) peak by proper intensity and at proper angle. True enough authors [8] by themselves notice for NiTi obvious proximity of the structure of the R-phase to the structure different from P3. But they suggest the $P \overline{3} 1 \mathrm{~m}$ as later for unknown reason.

\section{CONCLUSION}

Our approach was employed successfully in previous works to many crystals with different structures [1]. They have been shown to be very useful for the analysis of martensitic phase transitions and martensitic phase structures in NiTi. It is necessary to confirm a new model of trigonal phase by the fullprofile analysis of new neutron diffraction pattern which we suppose to obtained shortly. It is interesting to do it at several different temperatures in the range of existence of the trigonal phase. Besides it is desirable to retrace the positions and intensities of all superstructure reflections of type $1 / 3,1 / 3,0$.

The same examination is needed for our new monoclinic structure. Perhaps these two structures cannot be true simultaneously so far as their realization requires the different mechanism of the successive plane shears. But successful description of the orthorhombic structure B19 in our approach with the compensation mechanism is beyond doubt.

It is worth noting that the two our space groups $\mathrm{Bm}$ and $\mathrm{P} 31 \mathrm{~m}$ (for monoclinic and trigonal phases respectively) don't have the inversion center among other symmetry elements. In other words both symmetry groups are polar and hence they allow that the phases possess a certain physical property described by the macroscopic polar vector. More likely it is a vector of the deformation origin. This question can be related to the shape memory effect.

\section{References}

[1] Kassan-Ogly F.A., Naish V.E and Sagaradze I.V., Phase Transitions 49 (1994) 89-141

[2] Naish V.E., Novoselova T.V. and Sagaradze I.V., The physics of metals and mettalography 80, N.5 (1995) $485-506$

[3] Buhrer W., Gotthardt R. et al., Journal de Physique 43, N. 12 (Suppl.C4) (1982) 219 - 224

[4] Matthias R. and Deiseroth H.-J., Croatia Chemical Acta 68, N.4 (1995) $701-708$

[5] NaishV.E., Novoselova T.V. and Sagaradze I.V., The physics of metals and mettalography

[6] Ohba T., Emura Y. and Otsuka K., Material Transactions JIM 33, N. 1 (1992) 29 - 37

[7] Shapiro S.M., Metal. Transitions 12A, N.4 (1981) $567-573$

[8] Hara T., Ohba T. and Otsuka K., Journal de Physique 5 (Suppl.C8) (1995) 641 - 645 